\title{
Anastomoses Colônicas após Mucosectomia Química, em Ratos
}

\author{
Colonic Anastomosis after Chemical Mucosectomy in Rats
}

\author{
IVANA DUVAL-ARAUJO ${ }^{1}$; LUIZ EDUARDO MOREIRA TEIXEIRA ${ }^{2}$; RUBEM MATEUS C. MIRANDA ${ }^{3}$; \\ DAÍRTON MIRANDA ${ }^{4}$; CÍNTIA COUTINHO DE MORAIS ${ }^{5}$
${ }^{1}$ Professor associado-doutor, Faculdade de Medicina, Universidade Federal de Minas Gerais; ${ }^{2}$ Mestre. Aluno do Programa de Pós-Graduação em Cirurgia (Doutorado), Faculdade de Medicina, Universidade Federal de Minas Gerais; ${ }^{3}$ Médico; ${ }^{4}$ Médico Patologista; ${ }^{5}$ Médica.

DURVAL-ARAUJO I; TEIXEIRA LEM; MIRANDA RMC; MIRANDA D; MORAIS CC. Anastomoses Colônicas após Mucosectomia Química, em Ratos. Rev bras Coloproct, 2009;29(1): 065-070.

RESUMO: Objetivo: Avaliar, experimentalmente, os efeitos da mucosectomia química sobre a cicatrização de anastomoses colônicas. Metodologia: Estudou-se 17 ratos Wistar machos divididos nos seguintes grupos: A (n=12), anastomose colônica; B ( $n=13)$, anastomose colônica após mucosectomia. A mucosectomia foi realizada através da introdução de um bastão de nitrato de prata a $10 \%$, durante um minuto, através das duas bocas a serem anastomosadas, e as anastomoses realizadas em plano único total, evertente, com fio polivicril 6-0 em pontos separados. Os animais foram estudados após 7 dias (6 do grupo A1 e 6 do grupo B1) e 14 dias (7 do grupo A2 e 6 do grupo B2) da cirurgia, e realizadas observações macroscópicas da presença de aderências, fístulas (saída de secreção através da anastomose ou teste da pressão de ruptura igual a zero), estenose (dilatação intestinal proximal à anastomose), abscessos peri-anastomóticos e peritonite. Foi também avaliada a pressão de ruptura das anastomoses e histologia das anastomoses. Os resultados qualitativos foram avaliados pelo teste do Qui-quadrado (com correção de Yates) e os quantitativos através do teste de Kruskall-Wallis, sendo considerados significativos valores de p<0,05. Resultados: Nenhum dos animais estudados apresentou fístulas. A incidência de aderências peri-anastomóticas não foi diferente entre os grupos (A1=100\%, $\mathrm{B} 1=\mathbf{1 0 0 \%}$, p=N.S.; A2=75\%, B2=50\%, p=N.S.). Houve semi-obstrução da anastomose em $25 \%$ dos animais do grupo A1, $50 \%$ daqueles do grupo B1, $25 \%$ dos animais do grupo A2 e $50 \%$ dos animais do grupo B2, sem diferença significativa entre os grupos. A pressão de ruptura, em mmHg, foi de 27,0 \pm 4,5 no grupo $A 1,34,5 \pm 2,0$ no grupo $B 1,28,9 \pm 4,2$ no grupo $A 2$ e 24,0 $\pm 3,4$ no grupo B2, com aumento significativo no grupo $B 1$ quando comparados aos grupos $\mathrm{A1}(\mathrm{p}=\mathbf{0 , 0 3})$ e B2 (p=0,02). Concluindo, a mucosectomia química com o uso do nitrato de prata, ao contrário daquela realizada mecanicamente, não associou-se a maior incidência de complicações da anastomose no cólon direito de ratos. Houve, entretanto, aumento da força da anastomose no período inflamatório, sem influência no período de fibroplasia da cicatrização.

Descritores: Anastomoses Intestinais; Mucosectomia; Fístulas.

\section{INTRODUÇÃO}

A mucosectomia nas proctocolectomias por doenças inflamatórias intestinais e pólipos adenomatosos é utilizada com o objetivo de reduzir o risco de recidivas na linha de sutura após construção de reservatórios ileais. $(1,2,3,4,5)$. Entretanto, existem controvérsias quanto ao mérito de se realizar a mucosectomia, sendo que alguns estudos sugerem que a mucosectomia seguida de anastomose manual se associaria com pior resultado funcional do que quando realizada a anastomose mecânica sem a mucosectomia $(6,7)$.

$\mathrm{Na}$ cicatrização de segmentos do tubo digestivo, é conhecida a importância da submucosa como principal responsável pela aquisição de força tênsil (8), devido à presença de grande quantidade de células inflamatórias e fibroblastos, (9) sendo essas as células responsáveis pela síntese do colágeno $(10,11)$. Após uma mucosectomia, teoricamente essas superfícies 
estariam mais expostas e, portanto, a área sujeita à reação inflamatória seria mais extensa, podendo resultar não só em uma cicatriz mais forte, como também favorecer ao aumento na incidência de estenoses.

Observou-se, em um estudo clínico, que cerca de $47 \%$ dos pacientes submetidos às técnicas de confecção dos reservatórios ileais após mucosectomia apresentam complicações pós-operatórias, sendo que a maioria dessas (16\%) são decorrentes de obstrução intestinal (3). Outro estudo relatou cerca de $25 \%$ de incidência de abscessos perianastomoses nesse procedimento (12). Entretanto, não se sabe se essa reação cicatricial exacerbada pode ser pela exposição da submucosa ou pelo trauma provocado pela retirada da mucosa.

Em algumas situações clínicas, também, a mucosectomia pode ser tecnicamente difícil, como naqueles pacientes com colite ulcerativa grave, podendo então ser realizado o debridamento químico da mucosa por métodos variados, como hidróxido de sódio, formalina e nitrato de prata (13)

Com o objetivo de avaliar alterações cicatriciais no cólon de ratos após a mucosectomia química com nitrato de prata, realizou-se este estudo.

\section{MATERIAIS E MÉTODOS}

Foram estudados 25 ratos wistar fêmeas com idade variando entre 3 e 4 meses. Esses animais foram aleatoriamente distribuídos nos seguintes grupos experimentais: A1 $(n=6)$, anastomose colônica, sacrifício no sétimo dia do pós-operatório; B1 ( $\mathrm{n}=6)$, mucosectomia química, sacrifício no sétimo dia do pós-operatório; A2 $(\mathrm{n}=7)$, anastomose colônica, sacrifício no décimo-quarto dia do pós-operatório; B2 ( $\mathrm{n}=6)$, mucosectomia, sacrifício no décimo quarto dia do pós-operatório.

Os animais foram anestesiados através de xilazina $(10 \mathrm{mg} / \mathrm{Kg})$ associada a quetamina $(60 \mathrm{mg} /$ $\mathrm{Kg}$ ) IP. Após a anestesia, foi realizada laparotomia mediana e identificado o cólon ascendente, sendo feita sua secção, em um ponto distante da entrada de grandes vasos a cerca de $3 \mathrm{~cm}$ do ceco, utilizando-se tesoura cirúrgica. Após limpeza das duas bocas com algodão embebido em solução salina a $0,9 \%$, foi introduzido em cada boca bastão de nitrato de prata a $10 \%$ (Araujo Farmácia de Manipulação, Brasil) durante um minuto em cada abertura, envolvendo uma extensão de cerca de $0,5 \mathrm{~cm}$ de mucosa colônica. Após a verificação da necrose da mucosa, foi feita a sutura intesti- nal em plano único evertente com pontos separados de polivicril 6-0, sendo feitos cerca de oito pontos em cada anastomose. Nos animais dos grupos $\mathrm{A}$ e $\mathrm{C}$, foi adotado o mesmo procedimento, sendo que introduziu-se durante um minuto uma compressa de algodão embebida em solução salina a $0,9 \%$ durante um minuto no lugar do uso do bastão de nitrato de prata. Em seguida, a cavidade abdominal foi fechada em dois planos, sendo o primeiro, músculo aponeurótico, com sutura contínua de categute 5-0 simples e a segunda, a pele, com sutura contínua de seda 4-0.

Os animais foram mantidos em caixas separadas por grupos e, durante 48 horas, sua alimentação foi restrita a solução glicosada a $25 \%$ ad libitum. Após este período, e até o momento do sacrifício, os animais receberam água e ração comercial padrão para ratos.

Após o tempo pré-estabelecido de observação pós-operatória, os animais foram anestesiados e feita a abertura da cavidade abdominal através de uma incisão mediana. Foi feita a identificação da anastomose e registradas as alterações macroscópicas na linha da anastomose colônica. As alterações macroscópicas pesquisadas foram a presença e o grau das aderências $(+$, ausente; $2+$, poucas, frouxas; $3+$, intensas, frouxas; $4+$, aderências firmes), presença de abscessos perianastomose, presença de peritonite, presença de fístulas tamponadas, presença de dilatação de alças colônicas ou entéricas acima da linha da anastomose. Em seguida, foi identificada a aorta abdominal, que foi seccionada para morte do animal por exsanguinação.

Um segmento de cólon de aproximadamente 3 $\mathrm{cm}$, contendo em seu ponto médio a anastomose, foi retirado para estudo da pressão de ruptura, de acordo com método padronizado em nosso meio. Resumidamente, a pressão de ruptura dos segmentos é avaliada através de insuflação de ar em uma das bocas, sob fluxo constante, estando o segmento imerso em uma cuba contendo solução salina a $0,9 \%$. O cateter através do qual o ar é insuflado conecta-se também a um manômetro de mercúrio, fornecendo a pressão exercida pelo aparelho. A medida da pressão de ruptura é dada pelo valor registrado no momento da ruptura da alça, verificado pela presença do borbulhamento no líquido contido na cuba (14).

Os segmentos colônicos foram depois fixados em formol a $10 \%$, a partir dos quais obteve-se cortes histológicos corados pela hematoxilina-eosina e pelo tricrômico de Gomori. Essas lâminas foram avaliadas com relação à presença de mucosa colônica na linha 
de sutura, grau da reação inflamatória e avaliação qualitativa do colágeno na linha de sutura.

Os dados da macroscopia e da histologia foram comparados entre os dois grupos através do teste do Qui-Quadrado, com correção de Yates, sendo considerados significativos valores de $\mathrm{p}<0,05$. Os dados da pressão de ruptura das anastomoses colônicas foram comparados entre os grupos através do teste de Kruskall-Wallis, sendo considerados significativos valores de $\mathrm{p}<0,05$

\section{RESULTADOS}

Em relação à macroscopia, não se observou fístula tamponada em nenhum animal nos quatro grupos estudados. Houve aderências em 100\% dos animais do grupo A1, $100 \%$ dos animais do grupo B1,75\% dos animais do grupo A2 e 50\% dos animais do grupo B2. Não houve diferença significativa entre os grupos. Observou-se obstrução parcial da anastomose em 25\% das anastomoses do grupo A1, $40 \%$ das anastomoses do grupo B1, 25\% das anastomoses do grupo A2 e $50 \%$ das anastomoses do grupo B2. Não houve diferença significativa entre os grupos (Figura 1).

A pressão de ruptura, em $\mathrm{mmHg}$, foi de $27,0 \pm$ 4,5 no grupo $\mathrm{A} 1,34,5 \pm 2,0$ no grupo $\mathrm{B} 1,28,9 \pm 4,2$ no grupo A2 e 24,0 $\pm 3,4$ no grupo B2. Essa pressão foi maior nos animais do grupo B1 (com mucosectomia) quando comparados àqueles do grupo A1 (sem mucosectomia) no sétimo dia de pós-operatório ( $\mathrm{p}=0,03$ ), e foi maior também quando comparados os animais com mucosectomia no sétimo (grupo B1) e décimo-quarto (grupo B2) dias de pós-operatório $(\mathrm{p}=0,02)$. Não houve diferença entre as pressões de ruptura nos animais sem mucosectomia no sétimo (grupo A1) ou décimo quarto (grupo A2) dias de pós-operatórios, e nem entre os animais sem mucosectomia (grupo A2) e naqueles onde foi feita a mucosectomia (grupo B2, Figura 2).

À histologia, observou-se que a reação inflamatória foi mais exuberante nos animais do grupo B1, mas sem diferença entre aqueles do grupo A1, B2 e A2. Observou-se também regeneração parcial da mucosa nos animais do grupo B2.

\section{DISCUSSÃO}

A cicatrização das feridas é definida como um processo altamente regulado de várias sequiências ce- lulares, levando à integridade do tecido após a lesão. $\mathrm{O}$ processo da cicatrização de alças intestinais processase de maneira única no organismo, com características diferentes da pele e outros tecidos orgânicos, tanto pelas características da síntese do colágeno como da migração de fibroblatos (9). A síntese do colágeno, mediada pela presença dos fibroblastos na anastomose, é um dos fatores responsáveis pela aquisição da força tênsil capaz de tornar as cicatrizes mais fortes com o tempo e, aparentemente, essa ativação dos fibroblastos ocorre não somente na linha de sutura, mas ao longo de

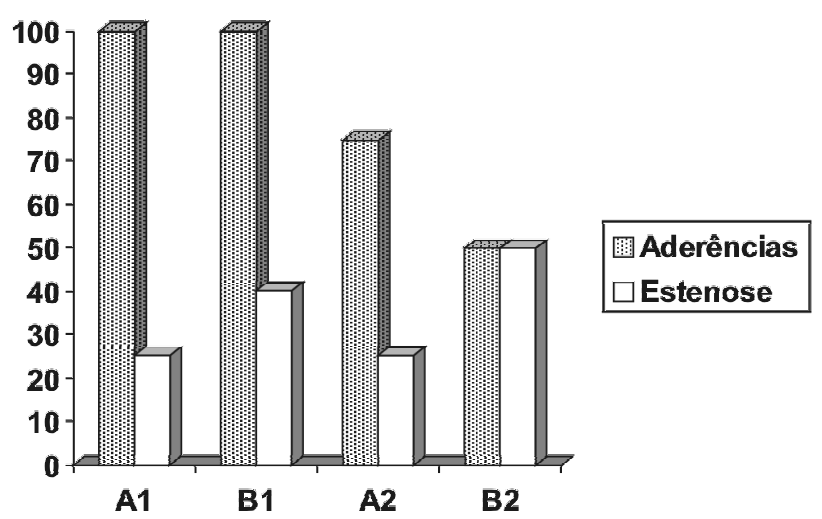

Figura 1 - Percentual de aderências e estenose parcial de anastomoses colônicas realizadas em ratos submetidos ou não a mucosectomia aos 7 e 14 dias de pós-operatório. Al (n=6), sem mucosectomia, anastomose com 7 dias; $B 1$ ( $n=6)$; com mucosectomia, anastomose com 7 dias; $A 2(n=7)$, sem mucosectomia, anastomose com 14 dias; $B 2(n=6) \mathrm{com}$ mucosectomia, anastomose com 14 dias.

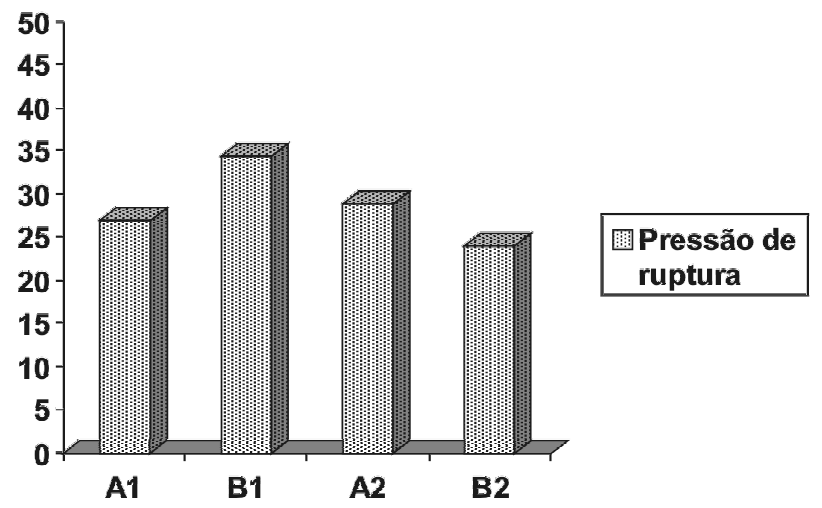

Figura 2 - Valor médio, em $\mathrm{mmHg}$, da pressão de ruptura de anastomoses colônicas realizadas em ratos submetidos ou não a mucosectomia aos 7 e 14 dias de pós-operatório. Al (n=6), sem mucosectomia, anastomose com 7 dias; $B 1$ ( $n=6)$; com mucosectomia, anastomose com 7 dias; $A 2(n=7)$, sem mucosectomia, anastomose com 14 dias; $B 2(n=6)$ com mucosectomia, anastomose com 14 dias. 
todo o trato digestivo $(10,11)$, caracterizando a importância da submucosa na cicatrização das anastomoses intestinais (8).

A confecção das anastomoses do tubo digestivo obedece a certas padronizações técnicas, visando reduzir a incidência de fístulas e aumentando sua segurança, como ausência de tensão na linha de sutura e ausência de hematomas (15). Certos detalhes técnicos, como sutura invertente ou evertente, em plano único ou em dois planos, persistem ainda como pontos controversos, havendo defensores tanto de uma quanto outra técnica.

Uma variação técnica que tem sido utilizada nos segmentos do tubo digestivo desprovidos de serosa é a retirada da mucosa, na técnica chamada de mucosectomia, onde a aposição das duas superfícies submucosas das bocas a serem anastomosadas permitiria, em tese, que fossem realizadas anastomoses com maior força tênsil, menos sujeitas, portanto, a ruptura $(14,16,17,18)$.

A mucosectomia tem sido empregada após as proctocolectomias e construção de reservatórios ileais em "J" ou em "S" com o objetivo de reduzir a incidência de recidiva das doenças inflamatórias e pólipos no segmento retal, e prevenindo a ocorrência de fístulas $(3,19)$. Segundo alguns relatos, a mucosectomia realizada nessas afecções aumenta a continência fecal e não promove aumento da morbidade associada à mucosa retal retida $(2,19)$. Existem diversas técnicas de mucosectomia descritas, mas a que parece associar-se a menor incidência de complicações é aquela que utiliza a indução da necrose da mucosa por meio químico, visto que o uso do bastão de nitrato de prata mostrou-se eficaz e factível em estudos experimentais (13), entretanto, alguns autores questionam se o grampeamento poderia ser superior à mucosectomia após as proctocolectomias na prevenção das complicações das anastomoses em presença da inflamação $(1,4,6,7)$, enquanto outros não demonstraram superioridade de uma técnica sobre outra (20). Entretanto, a mucosectomia se mostrou superior à sua não realização na prevenção da recidiva dos pólipos e doença inflamatória intestinal na linha de sutura (4)

Durante a cicatrização de alças intestinais, a eficácia e intensidade da resposta celular é o principal determinante da aquisição de força tênsil da sutura, prevenindo a formação de fístulas (21). Esses elementos celulares, presentes na submucosa, como macrófagos e fibroblastos, são os responsáveis pelo início e maturação do processo cicatricial $(9,21)$. Nas anastomoses em plano único, a superfície linear de contato entre as duas camadas submucosas das duas bocas anastomóticas forneceria a quantidade e ativação ideal dessas células, resultando na formação de uma cicatriz adequada (8). Entretanto, após uma mucosectomia, teoricamente essas superfícies estariam mais expostas e, portanto, a área sujeita à reação inflamatória seria mais extensa, podendo resultar não só em uma cicatriz mais forte, como também favorecer ao aumento na incidência de estenoses.

Observou-se, em um estudo clínico, que cerca de $47 \%$ dos pacientes submetidos às técnicas de confecção dos reservatórios ileais após mucosectomia apresentam complicações pós-operatórias, sendo que a maioria dessas (16\%) são decorrentes de obstrução intestinal (3). Há também o relato de uma maior incidência de abscessos perianastomoses nesse procedimento (12).

Em nosso estudo, observamos que, em relação à mortalidade, a técnica empregada não se associou a uma maior mortalidade operatória. À macroscopia, ao contrário do que se observa após a mucosectomia mecânica, não houve aumento significativo na incidência de aderências firmes e de estenoses entre os grupos. Entretanto, houve aumento da força das anastomoses no período inicial de fibroplasia (sétimo dia de pós-operatório) nos animais submetidos à mucosectomia, efeito que se perdeu na fase de maturação da cicatriz (décimo quarto dia de pós-operatório), sugerindo que na fase onde ocorrem as principais complicações clínicas nas anastomoses, a mucosectomia seria eficaz em sua redução.

À histologia, observou-se reação inflamatória mais exuberante no grupo de animais submetidos à mucosectomia e estudados no sétimo dia de pós-operatório (B1), os mesmos que apresentaram maior pressão de ruptura na anastomose. Tal fato sugere que a irritação promovida pelo nitrato de prata, associado à exposição da submucosa, podem ter intensificado a resposta inflamatória, sendo esta, talvez, a principal causa do aumento da força da anastomose. A regeneração parcial da mucosa nos animais do grupo B2, observada à histologia, difere dos dados obtidos por outros autores $(19,20)$. Entretanto, em nosso trabalho, não realizamos a retirada mecânica da mucosa necrosada, o que pode ter contribuído para que ilhas de mucosa viáveis remanescente tenham promovido a proliferação do epitélio intestinal, possibilitando sua regeneração. 
Concluindo, a mucosectomia química com nitrato de prata foi eficaz na remoção da mucosa, sem associar-se a complicações como deiscência da sutura ou estenose da anastomose no cólon de ratos.

\begin{abstract}
Objective: To evaluate, experimentally, the effects of chemical mucosectomy on colon healing in rats. Methods: We studied 17 male Wistar rats divided into following groups: A $(n=12)$, colonic anastomosis; $B(n=13)$, colonic anastomosis after use of $10 \%$ silver nitrate. The mucosectomy in group $B$ was made by means introduction of $10 \%$ silver nitrate pencil into anastomotic surfaces during 1 minute in an extension of $0.5 \mathrm{~cm}$. The anastomosis was made in single plane with evertent sutures of polyvicryl 6-0 in separated sutures. The animals were evaluated in the seventh postoperative day (A1, 6 of the group A and B1, 6 of the group B) and fourth postoperative day (A2, 7 of the group A and B2, 6 of the group B). The anastomosis was evaluated in macroscopic aspect (adherence, fistula, stenosis, perianastomotic abscesses, peritonitis), rupture pressure (mmHg) and histology (inflammatory infiltrate). The data were analysed by Chi-square test (qualitative data) and ANOVA (quantitative data), and considered significative values of $\mathbf{p}<\mathbf{0 . 0 5}$. Results: None animal presented fistula. The incidence of adherence was no different among the groups. $(\mathrm{A} 1=100 \%, \mathrm{~B} 1=100 \%, \mathrm{p}=\mathrm{NS} ; \mathrm{A} 2=\mathbf{7 5 \%}, \mathrm{B} 2=50 \%, \mathrm{p}=\mathrm{NS})$. There were anastomosis partial obstruction in $25 \%$ of group $\mathrm{A1}, 50 \%$ in $B 1,25 \%$ in $\mathrm{A} 2$ and $50 \%$ in $B 2$, without significant differences among the groups. The rupture pressure was $27,0 \pm 4,5$ in $A 1$ group, $34,5 \pm 2,0$ in $\mathrm{B} 1$ group, 28,9 \pm 4,2 in $\mathrm{A} 2$ group and 24,0 $\pm 3,4$ in group. There was an significant increase in pressure rupture in $\mathrm{B} 1$ group when compared with $\mathrm{A} 1$ group $(\mathrm{p}=\mathbf{0 , 0 3})$ and $\mathrm{B} 2$ group $(\mathrm{p}=\mathbf{0 , 0 2})$. Conclusion: The chemical mucosectomy with silver nitrate was not associated with increase in postoperative cicatricial complications in wound colon healing in rats. There was, however, an increase in anastomosis strength in early period of the cicatricial process, without influence in latter period.
\end{abstract}

Key words: Intestinal Anastomosis; Mucosectomy; Fistula.

\section{REFERÊNCIAS}

1. Dolgin, S.E.; Shlasko, E.; Benkov, K.; Leleiko, N. Restorative proctocolectomy in children with ulcerative colitis utilizing rectal mucosectomy with or without diverting ileostomy. J. Pediatr. Surg., 1999. 34:837-839.

2 Brasken, P.; Lehto, M.; Renvall, S. Changes in the connective tissue composition of the submucosal layer of colonic anastomosis. Acta Chir Scand., 1989. 155:413-419.

3. Lázaro da Silva, A.; Gontijo Filho, B. Sutura intestinal em plano único. Rev. Assoc. Med. Minas Gerais, 1974. 25:18-21.

4. Remzi, F.H.; Church, J.M.; Bast, J.; Lavery, I.C.; Strong, S.A.; Hull, T.L.; Harris, G.J.; Delaney, C.P.; O'r, M.G.; McGannon, E.A; Fazio, V.W. Mucosectomy vs. Stapled ileal pouch-anal anastomosis in patients with familial adenomatous polyposis: functional outcome and neoplasia control. Dis. Colon Rectum, 2001. 44:1590-1596.

5. Bauer, J.J.; Gorfine, S.R.; Gelernt, I.M.; Harris, M.T.; Kreel, I. Restorative proctocolectomy in patients older than fifty years. Dis. Colon Rectum, 1997. 40:562-565.

6. Chambers WM; McC Mortensen MJ Should ileal pouchanal anastomosis include mucosectomy? Colorectal Dis. 2007. 9:384-92

7. Silvestri MT; Hurst RD; Rubin MA Michelassi F; Fichera A Chronic inflammatory changes in the anal transition zone after stappled ileal pouch-anal anastomosis: is mucosectomy a superior alternative? Surgery 2008. 44:533-7

8. Martens M.F.W.C.; Huyben, C.M.L.C.; Hendriks, Th. Collagen synthesis in fibroblasts from human colon: regulatory aspects and differences with skin fibroblasts. Gut, 1992. 33:1664-1670.

9. Gemlo, B.T.; Belmonte, C.; Madoff, R.D. Functional assessment of ileal pouch-anal anastomotic techniques. Am. J. Surg., 1995. 169:137-141.

10. McMullen, K.; Hicks, T.C.; Ray, J.E.; Gathright, J.B.; Timmcke, A.E. Complications associated with ileal pouchanal anastomosis. World J. Surg., 1991. 15:763-766.

11. Taruffi, F.; Dannaoui, M.B.; Giovannini, F.; Cangioni, G. Jejunal mucosectomy as a preliminary technique of pancreatico-jejunal anastomosis. G. Chir., 1992. 13:217-218.

12. Aprilli, F.; Carril, C.F.; Guimarães, A.S.; Ferreira, A.L. Estudo comparativo da segurança de três tipos de anastomoses intestinais. Rev. Ass. Med. Bras., 1975. 21:307-308.

13. Kojima, Y.; Sanada, Y.; Fonkalsrud, E.W. Evaluation of techniques for chemical debridement of colonic mucosa. Surg. Gynecol. Obstet., 1982. 155:849-854.

14. Milagres LC; Duval-Araujo I; Barral SM; Grossi GC Efeito do uso de povidine-iodine na cicatrização de anastomoses de colon direito de ratos. Arch. Gastroenterol. 2005. 42:95-98

15. Ballantyne, G. The experimental basis of intestinal suturing. Dis. Colon Rectum, 1984. 27:61-72.

16. Barone, B.; Furlaneto, J.A.; Maranhão, R.F.A.; Goldenberg, S.; Oliveira, E. A submucosa e sua importância nas anastomoses em um plano. Estudo experimental em intestino grosso de cães. An. Paul. Med. Cir., 1979. 106:01-14.

17. Keighley, M.R. Abdominal mucosectomy reduces the incidence of soiling and sphincter damage after restorative proctocolectomy and J pouch. Dis. Colon Rectum, 1987. 30:386-390. 
18. Hawley, P.R.; Faulk, W.P.; Hunt, T.K.; Dunphy, J.E. Collagenase activity in the gastrointestinal tract. Br. J. Surg., 1970. 57:896-900.

19. Telander, R.L.; Perrault, J. Colectomy with rectal mucosectomy and ileoanal anastomosis in young patients. Its use for ulcerative colitis and familial poliposis. Arch. Surg., 1981. 116:623-629.

20. Schluender SJ; Mei L; Yang M; Fleshner PR Can metaanalysis answer the question: is mucosectomy and handsewn or double-stapled anastomosis better in ileal-pouch anastomosis? Am. Surg. 2006. 72:912-6
21. Whitaker, D.; Papadimitriou, J. Mesothelial healing: morphological and kinetic investigations. J. Pathol., 1985. 145:159-175.

\section{Endereço para correspondência:}

IVANA DUVAL DE ARAÚJO

Rua Bento Mendes Castanheira 121 / apto 102

CEP: $31260-270$

Belo Horizonte, Minas Gerais, Brasil 